\title{
A rare cause of bilateral pleural effusion - desmoplastic small round cell tumor
}

\author{
Archana Baburao $^{1}$, Poonam Maurya ${ }^{2}$, Amulya Chakenahalli³, Huliraj Narayanswamy ${ }^{3}$ \\ ${ }^{1}$ Department of Pulmonary Medicine, Rajarajeswari Medical College and Hospital, Bangalore; ${ }^{2}$ Department of medical \\ Oncology, Kempegowda Institute of Medical Sciences, Bangalore; ${ }^{3}$ Department of Pulmonary Medicine, Kempegowda \\ Institute of Medical Sciences, Bangalore, India
}

\begin{abstract}
Desmoplastic small round cell tumor (DSRCT) is a rare, extremely aggressive and malignant tumor predominantly affects young adolescent males and typically presents as a large intraabdominal mass. However, tumor arising from other body sites are also reported in the literature. Histology and immunohistochemistry play an important role in the diagnosis and differentiating this rare tumor from other round cell tumors. A multidisciplinary approach consisting of a combination of surgery, chemotherapy and radiation therapy is the treatment of choice as there is no standard therapy. We report a case of DSRCT of testis with pleural metastasis presenting
\end{abstract}

Correspondence: Dr Archana Baburao, Department of Pulmonary Medicine, Rajarajeswari Medical College and Hospital, Bangalore, India.

Tel. +91.9741016606

E-mail: dr_archana82@yahoo.co.in

Key words: Desmoplastic small round cell tumor; DSRCT; pleura; immunohistochemistry.

Ethics approval and consent to participate: No ethical committee approval was required for this case report by the Department, because this article does not contain any studies with human participants or animals.

Conflict of interest: The authors declare that they have no competing interests, and all authors confirm accuracy.

Contributions: $\mathrm{AB}, \mathrm{PM}$, designed case report; $\mathrm{AB}, \mathrm{AC}$, data collection; $\mathrm{PM}, \mathrm{HN}$, data analysis; AB, PM, AC, manuscript drafting. All the authors have read and approved the final version of the manuscript and agreed to be accountable for all aspects of the work.

Acknowledgements: The Authors would like to thank Dr. Savithri Ravindra (Pathologist) for providing the HPE slides.

Received for publication: 1 April 2021.

Accepted for publication: 6 October 2021.

CCopyright: the Author(s), 2021

Licensee PAGEPress, Italy

Monaldi Archives for Chest Disease 2022; 92:1874

doi: 10.4081/monaldi.2021.1874

This article is distributed under the terms of the Creative Commons Attribution Noncommercial License (by-nc 4.0) which permits any noncommercial use, distribution, and reproduction in any medium, provided the original author(s) and source are credited. as bilateral pleural effusion in a young adolescent male who was treated with both surgery and chemotherapy. However, the patient succumbed to illness after one year of diagnosis.

\section{Introduction}

Desmoplastic small round cell tumor (DSRCT) is a rare and highly aggressive neoplasm with characteristic histological, immunohistochemical pattern and chromosomal translocation features, first described as a distinct clinico-pathologic entity by Gerald and Rosai in 1989 [1]. These tumors predominantly occurs in young adolescent males and primarily occurs in the abdomen and pelvis [1,2]; however, tumor arising from other locations are also reported. The tumor possesses a diagnostic challenge due to its rarity and also due to the absence of specific clinical manifestations and similar morphologic appearances which it shares with other small round cell tumors. Prognosis is poor owing to advanced stage at presentation and lack of standard therapy. Only few cases of pleural involvement by DSRCT ( $<15$ cases) have been reported in the scientific literature. We report a case of DSRCT of testis with pleural metastasis presenting as bilateral pleural effusion.

\section{Case Report}

A 25-year-old male presented with dyspnea on exertion, left sided chest pain, fever with chills and dry cough for three weeks. He had lost three kgs of weight in one month and was an occasional smoker. On examination, he had tachypnea, tachycardia and room air saturation was $92 \%$. Head to toe examination was normal except for a right testicular swelling which the patient had not noticed. Respiratory system examination revealed absent breath sounds over bilateral infrascapular regions. Other systemic examination was normal. Routine blood investigations including complete blood picture, liver and renal function tests were normal. Arterial blood gas (ABG) showed type 1 respiratory failure. Chest $\mathrm{X}$-ray of the patient on day 1 showed bilateral pleural effusion (Figure 1a) and ultrasound abdomen showed enlarged retroperitoneal lymph nodes. Therapeutic pleural fluid aspiration was done on right side and $300 \mathrm{ml}$ of straw-colored fluid was aspirated and analysis revealed exudative fluid with high adenosine deaminase (ADA) levels and negative malignant cytology. Thorax CT (Figure 1b) and abdomen done on day 4 showed right mild and left gross pleural effusion, borderline hepatosplenomegaly, heterogeneously enhancing nodal mass in retroperitoneal region encasing and anteriorly displacing aorta (Figure 1c), inferior vena cava and 
branches of aorta and a right testicular mass lesion (Figure 1d). As there was high degree of suspicion for malignancy, $\beta$ HCG and Alpha fetoprotein were done which were within normal limits. Patient underwent right high orchidectomy. Even as the above reports were expected, patient became more breathless and had recurrent pleural effusion for which needle biopsy of pleura using Abram's needle was done followed by pigtail insertion, later pleurodesis using $100 \mathrm{ml}$ of $2 \%$ iodopovidone on left side. Histopathology of testicular mass revealed features of small round cell tumor (Figure 2a). Left pleural biopsy also revealed metastatic small round cell tumor (Figure $2 \mathrm{~b}$ ). Immunohistochemistry of pleura (Figure 3) was strongly positive for desmin and focal positivity for neuron specific enolase (NSE) and Wilms tumor (WT1). FISH (fluorescence in situ hybridization) analysis was negative for EWSR1 (22q12) gene translocation. A diagnosis of DSRCT of testis with pleural metastasis was made.

Patient was started on alternate regimen of IV vincristine, adriamycin, cyclophosphamide and ifosfamide, etoposide every three weeks. Plan was to complete 17 cycles. PET scan showed near complete response at the end of sixth cycle. However, at the end of 14 cycles, he developed ascites and obstructive jaundice. Repeat PET scan showed features of progressive disease and he succumbed to death one year after diagnosis.

\section{Discussion}

Desmoplastic small round cell tumor is an extremely rare and highly aggressive malignancy, with an estimated incidence is $0.2-$ 0.5 per million people. These tumors predominantly occurs in young males in their second or third decade of life, male $>$ female $(5: 1)$ predilection. It typically presents as a large intra-abdominal mass originating from the abdominal cavity and pelvis; thus, the retro peritoneum, omentum, and mesentery are often involved. Although extra abdominal DSRCT is rare, invasion of extra peritoneal sites like testis, ovaries, lung, pleura, intracranial, soft tissue and bone, parotid gland are also reported $[3,4]$. DSRCT needs to be differentiated from other round cell tumors (Table 1).

Invasion of pleura by DSRCT is extremely rare and challenging to diagnose, because of their rarity and unspecific demographic, clinical, and radiological features. It commonly presents with chest pain, dyspnea, cough and back pain. Different radiological manifes-

Table 1. Differential diagnosis of small round cell tumor.

\begin{tabular}{l} 
Ewing's sarcoma/peripheral neuroectodermal tumor \\
Neuroblastoma \\
\hline Rhabdomyosarcoma \\
Wilm's tumor \\
\hline Non-Hodgkin's lymphoma \\
Synovial sarcoma \\
Desmoplastic small round cell tumor \\
Small cell carcinoma
\end{tabular}

Retinoblastoma

Hepatoblastoma

Nephroblastoma

Small cell osteogenic sarcoma

Granulocytic sarcoma tations of pleural invasion by DSRCT include diffuse irregular or nodular pleural thickening, unilateral or bilateral pleural effusion [4], multiple pulmonary nodules or solid mass in thorax.

Among the extra abdominal metastases of DSRCT, Bellah et al. [5] and Biswas et al. [6] reported pleural effusion in only two out of 11 patients and one out of 18 patients respectively. In majority of previously reported cases of pleura invasion by DSRCT, left pleura

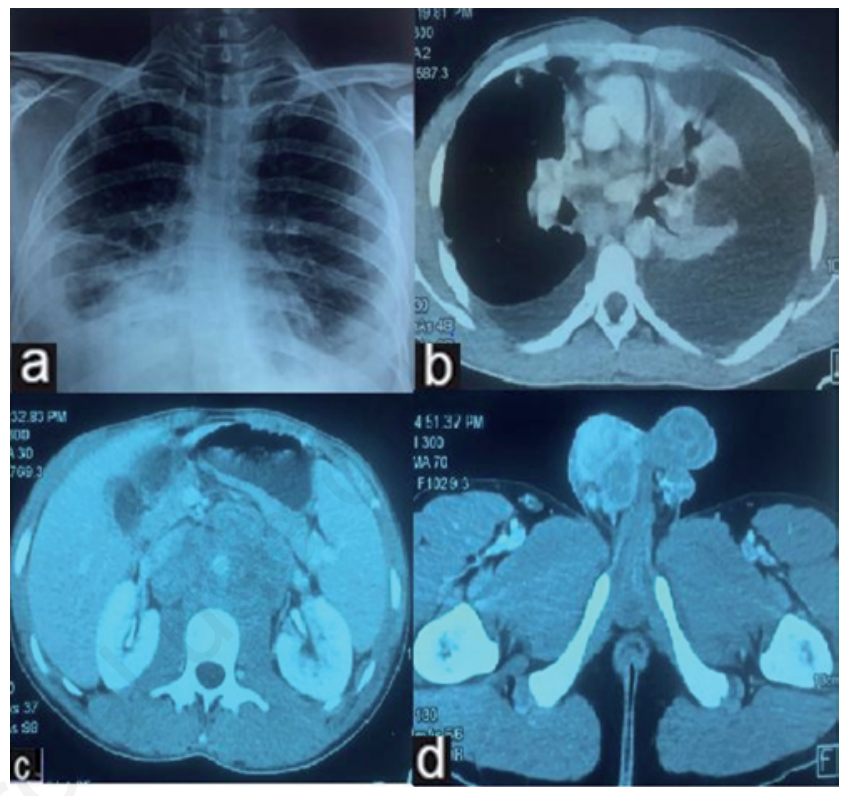

Figure 1. a) Chest $\mathrm{X}$ ray showing bilateral pleural effusion; b) axial cut section $\mathrm{CT}$ thorax showing bilateral pleural effusion $\mathrm{L}>\mathrm{R}$; c) CT abdomen showing heterogeneously enhancing nodal mass in retroperitoneal region encasing and anteriorly displacing aorta; d) CT pelvis showing large right testicular mass lesion.

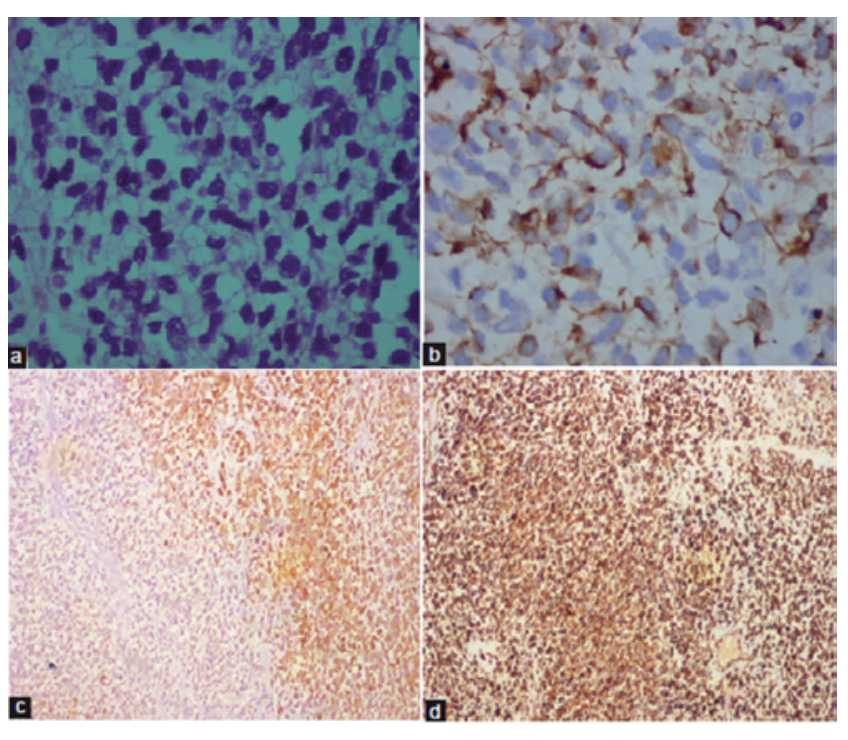

Figure 2. a) Pleural biopsy showing small round blue cells separated by abundant desmoplastic stroma (H\&E staining $x 100)$; b) immunohistochemical positivity for desmin in pleural biopsy; c) positivity for neuron-specific enolase (NSE); d) positivity for WT1. 
was involved in $46.7 \%$, right pleura in $26.7 \%$ and bilateral in $20 \%$ of the cases in the form of pleural effusion [7]. Similarly in a study involving 60 patients of abdominal DSRCT, pleural effusion was the presentation only in $5 \%$ of patients and also pleural effusion was identified as a potential risk factor for adverse outcome [8].

It has been suggested that this tumor may be derived from the primitive mesothelium or submesothelial mesenchyme. Characteristic histologic features of DSRCT include well-defined nests composed of small round cells, separated by abundant desmoplastic stroma. A definite diagnosis can be made with a demonstrated multidirectional differentiation from other round cell tumors and coexpression of epithelial [cytokeratin (CK) and epithelial membrane antigen (EMA)], mesenchymal (vimentin and desmin) and neural (CD56 and NSE) markers [9]. A distinct staining pattern for desmin, namely the punctuate and perinuclear cytoplasmic positivity is seen. Almost all DSRCTs are positive for WT1 genes. Cytogenetic study shows unique (11:22), (p13:q12) translocation which results in an active fusion protein involving the Ewing sarcoma (EWS) and WT1 genes, which was present in 29 out of 32 tumors in a study by Lae et al. [9]. EWSR1/WT1 fusion gene has shown to induce the upregulation of platelet-derived growth factor (PDGF) ligand and receptors, which might be responsible for the excessive production of desmoplasia in DSRCT.

There is no standard therapy for DSRCT. A multidisciplinary approach consisting of a combination of surgery, chemotherapy and radiation therapy is adopted in most cases in literature. Whole abdomino-pelvic irradiation (WAPI) as a novel approach for the residual disease following aggressive chemotherapy and debulking surgery has been reported with overall survival and relapse free survival rate of $48 \%$ and $19 \%$ respectively at the end of three years [10]. The most recent chemotherapy regimen employed is the "P6 protocol", which consists of cyclophosphamide, doxorubicin, vincristine, etoposide and ifosfamide [11].

Several other novel agents such as tyrosine kinase inhibitors pazopanib, sunitinib, anti-VEGF agent - bevacizumab, insulin growth factor-1 receptor (IGF-1R) antibody - ganitumab, cixutumumab, mammalian target of rapamycin (mTOR) inhibitor - temsirolimus have all been reported to be well tolerated and having good antitumor activity in patients with pre-treated DSRCT. With the available best treatment modality, the overall survival rate is less than $44 \%$ at three years and only $15 \%$ at five years [12].

\section{References}

1. Gerald WL, Rosai J. Case2. Desmoplastic small round cell tumor with divergent differentiation. Paediatr Pathol 1989;9:177-83.

2. Ordonez N, Zirkin R, Bloom RE. Malignant small-cell epithelial tumor of the peritoneum expressing mesenchymal-type inter-mediate filaments. Am J Surg Pathol 1989;13:413-21.

3. Thuret R, Renaudin K, Leclere J, et al. Uncommon malignancies: Paratesticular desmoplastic small round-cell tumor. J Clin Oncol 2005;23:6253-5.

4. Parkash V, Gerald WL, Parma A, et al. Desmoplastic small round cell tumor of the pleura. Am J Surg Patho. 1995;19:659-65.

5. Bellah R, Suzuki-Bordalo L, Brecher E J, et al. Desmoplastic small round cell tumor in the abdomen and pelvis: report of CT findings in 11 affected children and young adults. AJR Am J Roentgenol 2005;184:1910-4.

6. Biswas G, Laskar S, Banavali SD, et al. Desmoplastic small round cell tumor: extra abdominal and abdominal presentations and the results of treatment. Indian J Cancer 2005;42:78-84.

7. Fois A.G, Pirina P, Arcadu A, et al. Desmoplastic small round cell tumors of the pleura: a review of the clinical literature. Multidiscip Respir Med 2017;12:22.

8. Scheer M, Vokuhl C, Blank B, et al. Desmoplastic small round cell tumors: Multimodality treatment and new risk factors. Cancer Med 2019;8:527-42.

9. Lae ME, Roche PC, Jin L, et al. Desmoplastic small round cell tumor: A clinicopathologic, immunohistochemical, and molecular study of 32 tumors. Am J Surg Pathol 2002;26:823-35.

10. Goodman KA, Wolden SL, La Quaglia MP, Kushner BH. Whole abdomino-pelvic radiotherapy for desmoplastic small round cell tumor. Int J Radiat Oncol Biol Phys 2002;1:170-6.

11. Watanabe T, Miyamoto S, Kitagori K, et al. A case of long-term survival of metastatic desmoplastic small round cell tumor treated with multimodal therapy. Oncol Lett 2012;3:30-4.

12. Lal DR, Su WT, Wolden SL, et al. Results of multimodal treatment for desmoplastic small round cell tumors. J Pediatr Surg 2005;40:251-5. 